\title{
A Study on Accelerated Thermal Aging of High Modulus Carbon/Epoxy Composite Material
}

\author{
Kyung Ju Min ${ }^{\mathrm{a}}$, Ji-Ung Choi ${ }^{\mathrm{b}+}$ and Ho-Sung Lee $\mathrm{e}^{\mathrm{a}, \mathrm{b}^{*}}$ \\ ${ }^{a}$ Korea Aerospace Research Institute \\ 169-84 Gwahak-ro, Yuseong-gu, Daejeon 34133, Korea \\ ${ }^{b}$ Department of Space Launch Vehicle System Engineering \\ University of Science \& Technology, 217 Gajung-dong Yuseong-gu, Daejeon 305-350, Korea, \\ ${ }^{+}$currently at Gwangju Institute of Science and Technology, Gwangju 500-712, Korea
}

\begin{abstract}
Composite materials have been used increasingly for various space applications due to the favorable characteristic of high modulus to density ratio and potential for near-zero coefficient of thermal expansion. In composite system, depending on the orientation of fibers, strength and stiffness can be changed so that the optimum structure can be accomplished. This is because the coefficient of thermal expansion (CTE) of carbon fibers is negative. For spacecraft and orbiting space structure, which are thermally cycled by moving through the earth' shadow for at least 5 years, it is necessary to investigate the change of properties of the material over time. In this study, thermal aging of epoxy matrix/high modulus carbon fiber composite materials are accelerated to predict the long term creep property. Specimens are tested at various temperatures of $100 \sim 140^{\circ} \mathrm{C}$ with dynamic mechanical analysis to obtain creep compliances that are functions of time and temperature. Using Time Temperature Superposition method, creep compliance curves at each temperature are shifted to the reference temperature by shift factor and a master curve is generated at the reference temperature. This information is useful to predict the long term thermal aging of high modulus composite material for spacecraft application.
\end{abstract}

\section{Introduction}

Polymer matrix composite(PMC) materials play a key role in lightweight spacecraft structure[1] with high specific stiffness (modulus-to-density ratio), corrosion resistance, design flexibility, reducing number of assemblies and fasteners, and high resistance to fatigue damage. In addition, carbon fiber composite structure can show the coefficient of thermal expansion(CTE) close to zero, since the CTE of highly aligned carbon fibers between 0 and $500{ }^{\circ} \mathrm{C}$ is negative while as that of the epoxy matrix is positive. By proper arrangement of reinforced fiber, the optimum condition for dimensional stability can be achieved. This is important since the mechanical and dimensional stability during transport into orbit and flight through the earth' shadow is required for an often lengthy mission. Application of composite includes spacecraft busses, antennas and optical benches. Therefore, the long-term performance of composite materials must be investigated to understand the degradation of properties during the intended service life of the spacecraft[2]. In space environment, the degradation of polymer matrix composites comes from radiation, temperature, thermal cycling, atomic oxygen, micrometeoroids, and contamination.
In this paper, thermal aging of epoxy matrix/high modulus carbon fiber composite materials are accelerated to predict the long term creep property. Generally most of polymer matrix composites exhibit poor thermal conductivity, the strength of composite materials have been widely considered for aircraft. However, for spacecraft, the stiffness and thermal conductivity must be considered for thermal management like heat pipe panels of satellites. High modulus pitch based carbon fibers are currently available with high thermal conductivity for satellite. Specimens are tested at various temperatures of $100 \sim 140^{\circ} \mathrm{C}$ with dynamic mechanical analysis to obtain creep. Creep compliance curves at each temperature are used with Time Temperature Superposition method to predict the long term creep compliance change. Even though the creep behavior of composite material is sensitive to various space environment, the creep in this study is accelerated by temperature change while other variables are kept constant.

\section{Experimental}

The composite prepreg was made of YS90A carbon fiber(NGF Co.) with 6,000 fibers per tow and RS3232 resin(HFG Co.). The YS90A fiber is a continuous pitch based fiber, which shows very high thermal

akjmin@kari.re.kr, ${ }^{\mathrm{b}}$ jiungchoi@ust.ac.kr, ${ }^{\text {}}$ hslee@kari.re.kr 
conductivity $(500 \mathrm{~W} / \mathrm{m}-\mathrm{K})$ and high modulus of $127 \mathrm{Msi}$. This material is appropriate for spacecraft, which requires multi-function of stiffness and thermal properties in space environment. In the heat rejection system of satellite, electronic devices producing heat are mounted directly on to structural composite sandwich panel. The electronic devices in spacecraft tend to be located close possible to each other reducing size and weight, which has resulted in a need for high thermal conductivity due to the increase in device density generates more heat per unit volume. Therefore, the electronic panel must dissipate the waste heat effectively to keep device temperature within the specified limits ensuring reliable operation. The composite specimens were characterized by measuring resin content, volatile content, DMA(Dynamic Mechanical Analysis), TMA(Thermo-Mechanical Analysis), and DSC(Differential Scanning Calorimetry). The size of the specimen is $2.0 \mathrm{~mm} \times 2.5 \mathrm{~mm} \quad \mathrm{x} \quad 22 \mathrm{~mm}$ (thickness $\times$ width $\times$ length) for Perkin Elmer DMA7. The glass transition temperature was obtained with Perkin Elmer Pyris-1 DSC and DMA7 with TMA mode. The creep test was performed on Perkin Elmer DMA7 with 3-point bending mode. An experimental study was reported the consistency in DMA flexure creep tests and tensile creep tests[3]. The creep compliance and storage modulus of epoxy resin were obtained as the viscoelastic modulus. Three point bending creep tests for the composite were carried out under various temperatures using DMA.

The creep compliance was measured with a constant force of $110 \mathrm{mN}$ for 3 hours. After forcing the specimen underwent recovery process and this procedure was repeated for temperature ranges of $100 \sim 140^{\circ} \mathrm{C}$ as shown in Fig. 1.

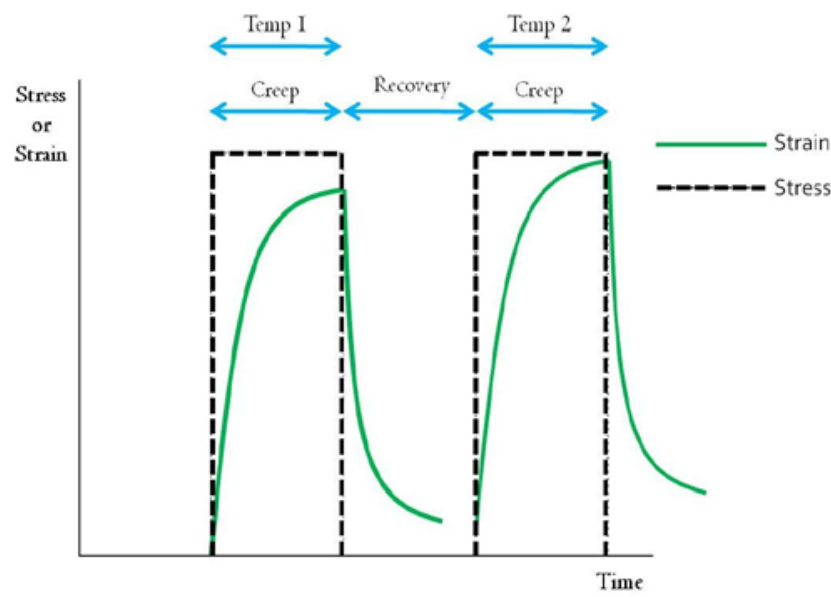

Figure 1. Creep sequenced test procedure [4]

\section{Results and discussion}

The theoretical background about Time Temperature Superposition for viscoelastic behavior is well established[2,4,5]. The dynamic mechanical behavior and storage modulus of the composite material was shown in Fig. 2. The quantity E', Young's storage modulus, is a measure of the energy stored elastically, whereas E", Young's loss modulus, is a measure of the energy lost as heat. The quantity $\tan \delta$ is calculated by E"/E' and called the loss tangent, $\delta$ being the angle between the in-phase and out-of-phase components in the cycle motion. The maximum in $\tan \delta$ is used for the glass transition temperature $(T g)$.

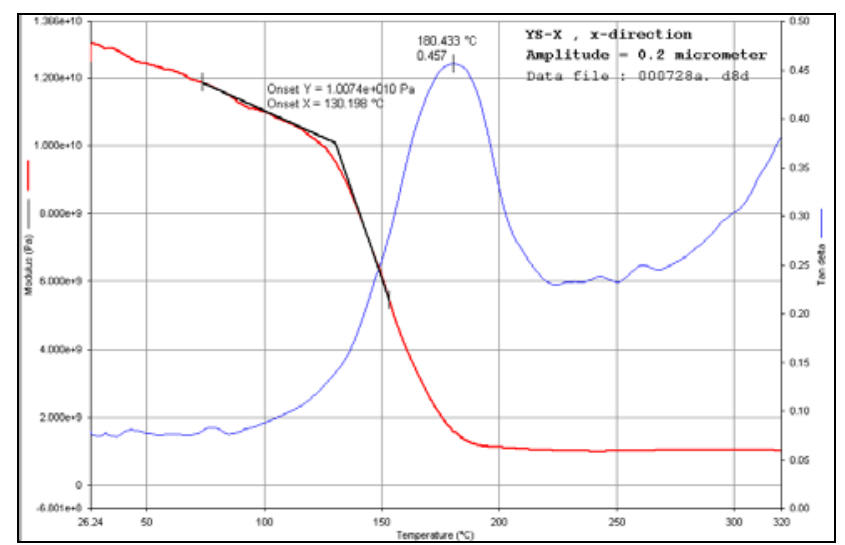

Figure 2. DMA curve for $T g$

Fig. 3(a) shows the result of creep compliance at different temperatures in $\log$ scale. When the property functions at various temperatures are shifted along the logarithmic time axis, they can be brought into a superimposed form. As in Fig. 3(b), curves at various temperatures can be shifted with respect to the reference temperature to form an extended curve to long-term range along the $\log$ (time) axis. The extended curve is called a master curve.

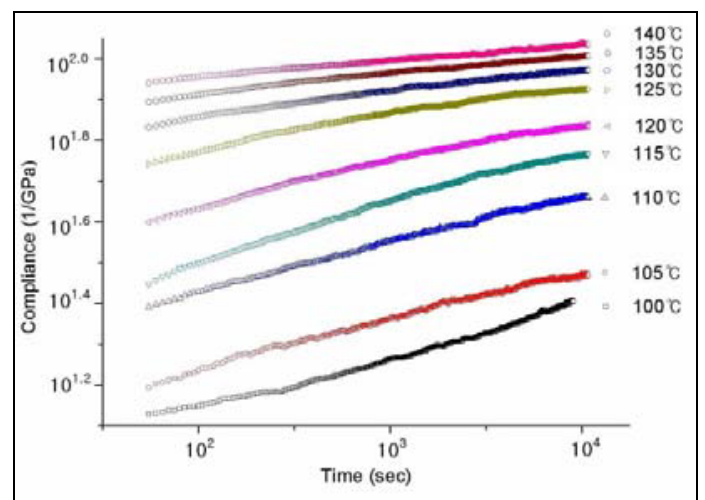

(a)

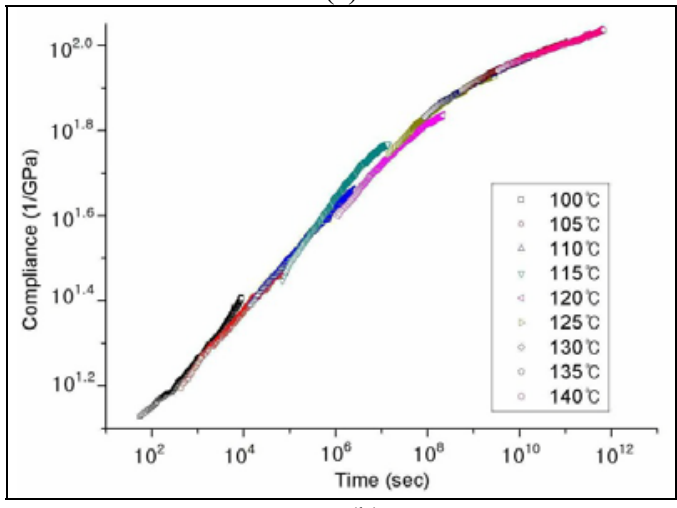

(b)

Figure 3. (a)Creep compliance at different temperatures in log scale, and

(b) master curve at a reference temperature of $100^{\circ} \mathrm{C}[6]$ 
The ratio between the original time $(t)$ and the shifted time $\left(t^{\prime}\right)$ is mainly a function of temperature and is called a time-temperature shift factor. The shift factors can be correlated with the temperature by Arrhenius equation [5] as follows:

$$
\log a_{T}=\frac{\Delta H}{2.303 R}\left(\frac{1}{T}-\frac{1}{T_{\text {ref }}}\right)
$$

where $\Delta H$ is the activation energy, and $R$ is the gas constant. The shift factors can be fit linearly on an inverse of temperature scale. The activation energy is a measure of energy barrier that must be overcome when molecular motion of the viscoelastic material is to occur. This method is valid when the same mechanism is present over the temperature range and the activation energy should be independent on temperature. In this temperature range, the activation energy is $52 \mathrm{KJ} / \mathrm{mol}$.

Fig. 4 shows the time-temperature shift factors for a reference temperature of $100^{\circ} \mathrm{C}$. Using this information it is possible to predict the long term thermal aging of pitch based composite material for spacecraft application. For instance, the decrease in modulus after 5 years is determined to be about $20 \%$.

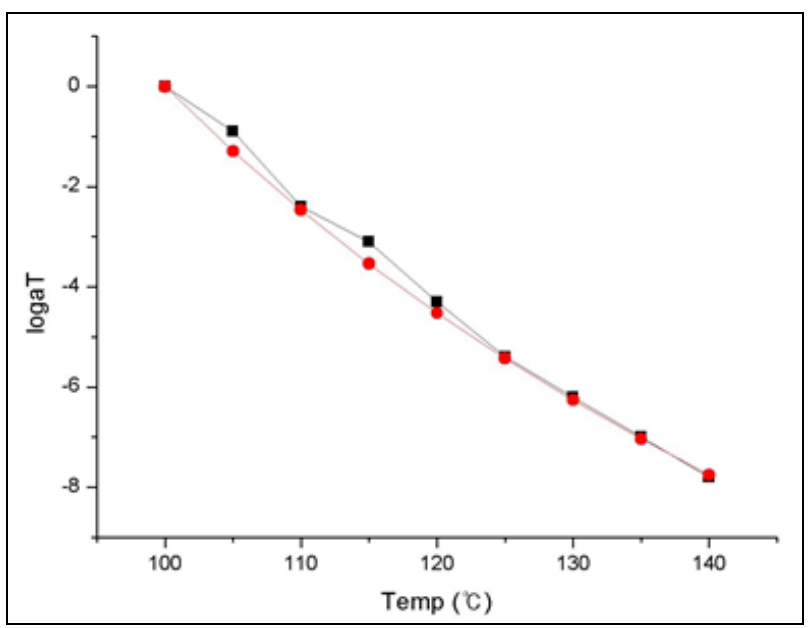

Figure 4. Time temperature shift factor for a reference temperature of $100^{\circ} \mathrm{C}[6]$

\section{Conclusion}

The results presented show the benefits of using time temperature superposition to accelerate the thermal aging of spacecraft composite material with pitch based carbon fiber. The shift factor for a reference temperature of $100^{\circ} \mathrm{C}$ is obtained and it is found that the modulus decreases to $80 \%$ of the original value.

\section{References}

1. E. M. Silverman, Technology Review Journal. Fall/Winter 2005, pp.1-19.

2. K. G. N. C. Alwis. and C.J. Burgoyne, Appl. Compos. Mater., 13(2006), pp. 249-264.

3. B. M. Becky and T. S. Gates, NASA Technical Report 2004008600, January 1, 2000.

4. B.M. Abdel-Magid and T.S. Gates, AIAA Paper 2003-4181.

5. J. R. Reeder, NASA TM-2001-2-211781.

6. J. U. Choi, A Study on Prediction of Long-term Creep Compliance of Pitch-based Composite using Time-Temperature Superposition Time-Temperature Superposition, M.S. Thesis, Feb. 2014, University of Science \& Technology at Korea. 